\title{
Letter to Editor \\ PTSD Symptoms and Risk Factors During the COVID-19 Disease Pandemic in Iran
}

\author{
Seyyed Mohammad Hossein Javadi ${ }^{1}$, Roya Marsa ${ }^{2}$ (), *Fahimeh Rahmani ${ }^{2}$ (1)
}

1. Department of Social Work, University of Social Welfare and Rehabilitation Sciences, Tehran, Iran. 2. Department of Counseling, University of Social Welfare and Rehabilitation Sciences, Tehran, Iran.

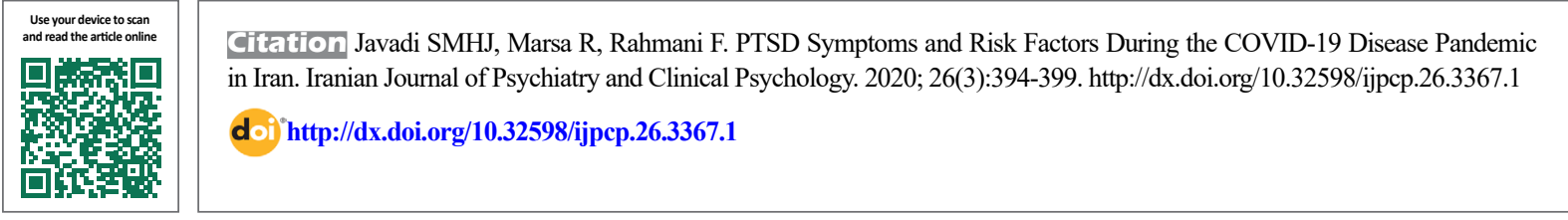

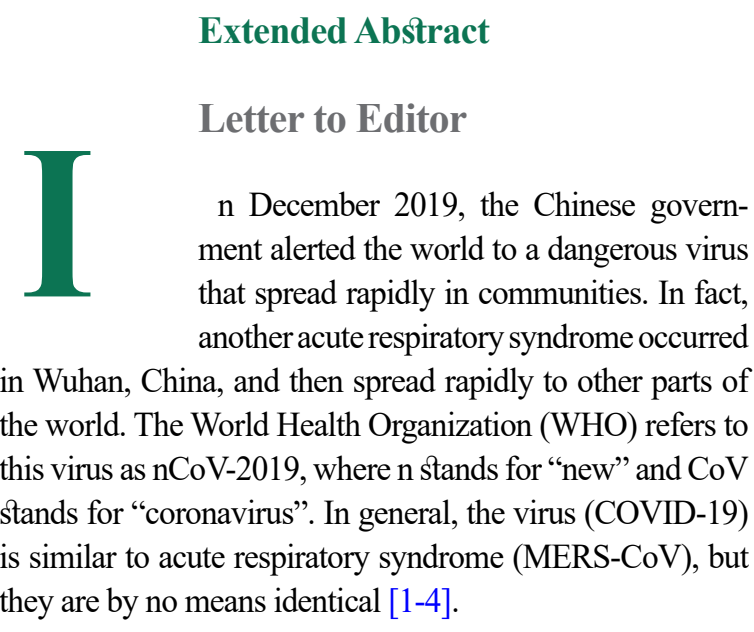

On March 11, the WHO declared the outbreak of COVID-19 an epidemic. According to the latest information from this organization, more than 2.39 million infections have been registered globally, and more than 162 thousand people have died [5]. Due to the pandemic and the spread of COVID-19 in just a few weeks, the WHO has been deeply concerned about this high prevalence and transmission rate, addressing it as a crisis and an emergency plan of the $\mathrm{WHO}[6]$.

However, at the core of any physical discomfort lies a strong connection between social, psychological, and physiological concepts. The main subject of any illness report is a person whose physiological function has changed and is out of balance - those who may be subject to psychological constraints and whose social relationships are seriously threatened [7]. In addition to physical and physiological discomfort, coronavirus disease also causes psychological problems such as mental disorders due to fear of transmitting the virus to others, loneliness, insomnia, home quarantine, reduced social relationships, disruption of everyday life, and so on [8].

As COVID-19 progresses, the clinical symptoms and psychological problems of those with the disease will worsen. Many will experience some degree of anxiety disorder and depression even after recovering and being discharged from the hospital [8-10].

Another psychological disorder that occurs in the face of events such as the traumatic experience of dealing with a life-threatening viral infection is Post-Traumatic Stress Disorder" (PTSD). According to the Diagnostic and Statistical Manual of Mental Disorders -Fifth Edition (DSM-5), PTSD includes a set of symptoms that follow a traumatic stressor and usually involve a perceived threat to one's life (one's own life or another person's life) or one's physical integrity, extreme fear, helplessness or panic [11, 12].

Studies had indicated that when the Severe Acute Respiratory Syndrome (SARS) epidemic led to PTSD, the symptoms were very similar to those experienced in severely stressful situations such as a terrorist attack or an earthquake. A high proportion of the Hong Kong population showed moderate to severe symptoms of PTSD between 2003 and 2004 following the outbreak of SARS.

* Corresponding Author:

Fahimeh Rahmani, PhD.

Address: Department of Counseling, University of Social Welfare and Rehabilitation Sciences, Tehran, Iran.

Tel: +98(21) 33401220

E-mail: fahimehrahmani@ymail.com 
According to the results of some studies on the mental health of medical staff in 2003 at the time of the SARS outbreak, about $1 \%$ of participants reported high levels of PTSD. A study of the rate of chronic psychiatric disorder among SARS patients showed that PTSD was the most common chronic psychiatric disorder (the cumulative incidence of PTSD within 30 months of the onset of SARS was approximately $47.8 \%$ ). In another study, about $44.1 \%$ of SARS patients received a diagnosis of PTSD within 2 to 46 months. According to these studies, follow-up and initial interventions for PTSD disorder during the outbreak of lifethreatening physical illness seem necessary $[6,13]$.

According to a new study by Sun et al. (2020), about one month after the pandemic and the outbreak of COVID-19, about $4.6 \%$ of the participants had experienced high levels of PTSD. Many factors such as "female gender", "living in a city with COVID-19", "poor sleep quality", and "previous experience of exposure to a dangerous and pervasive phenomenon" were significantly associated with PTSD severity. But there was no significant relationship between PTSS rate and "age" and "education" $[6,12]$. According to studies, it seems necessary that high-risk factors in the prevalence of PTSD, such as "female gender" (who usually experience high levels of anxiety and depression), "the quality of sleep", "living in cities with COVID-19", etc. to be considered.

Due to the possible prevalence of PTSD in COVID-19 patients who also have risk factors such as "female gender", late psychosocial interventions can lead to chronic, persistent, and stabilized symptoms and severe psychological reactions. Therefore, crisis management in this group of people and reducing this crisis's dimensions will not be possible except concerning all dimensions and aspects of human issues.

During the COVID-19 crisis, this group of patients experiencing such traumatic events need adequate social support. Some of the psychological treatment approaches used during the SARS outbreak can treat COVID-19 psychiatric emergencies. In fact, as traditional psychiatric services (such as face-to-face counseling) are not available during the $\mathrm{CO}$ VID-19 epidemic, therapists focus on online psychological services. The International Society for Traumatic Stress Studies (ISTSS) and the American Psychiatric Association (APA, 2017) have suggested that therapists regularly assess patients' mental health status after their physical condition stabilizes. They should also use evidence-based and costeffective approaches (cognitive-behavioral therapy, cognitive process therapy, and exposure therapy) for PTSD patients [14-16].
Studies have revealed that crisis intervention must be dynamic and adapt to the different stages of the COVID-19 epidemic (during and after the outbreak). Health professionals should be actively involved in the patient intervention process during the outbreak. In this process of intervention, two activities must be performed simultaneously: 1. Intervention for fear of illness, which is mostly done by doctors and with the help of psychologists; 2 . Intervention for the patient's inability to adapt to critical situations, which is done mainly by social psychologists. But severe psychological problems such as violence and suicide are managed by a psychiatrist [17].

In general, mental health professionals and social workers, on the one hand, by providing a variety of interventions tailored to the needs of this group of patients, and on the other hand, by addressing their psychosocial issues through social networks and telephone counseling, can reduce the anxiety and psychological distress of these patients. As a result, providing psychosocial-medical interventions and training can cause psychological and social adjustment, improve mental health, and ultimately prevent the complications of psychological problems caused by this crisis [18].

Therefore, the need to design and develop short-term and unattended psychosocial interventions and protocols for vulnerable groups becomes more apparent.

\section{Ethical Considerations}

\section{Compliance with ethical guidelines}

This study ethically was approved by University of Social Welfare and Rehabilitation Sciences.

\section{Funding}

This research did not receive any grant from funding agencies in the public, commercial, or non-profit sectors.

\section{Authors' contributions}

All authors contributed equally in preparing all parts of the research.

\section{Conflicts of interest}

The authors declared no conflicts of interest. 
This Page Intentionally Left Blank 


\title{
نشانهها و عوامل خطرساز اختلال استرس يس از سانحه در طول ياندمى شدن كوويد-9 ( در ايران
}

\author{
سيد محمد حسين جوادى'، رويا مرسا؟ ه. "فهيمه رحمانى' (1) \\ 1. ا.كروه مددكارى اجتماعى، دانشكاه علوم بهزيستى و توانبخشى، تهران، ايران. \\ r. كروه مشاوره، دانشكاه علوم بهزيستى و توانبخشي، تئران، ايران.
}

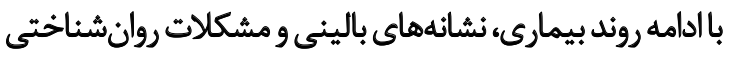

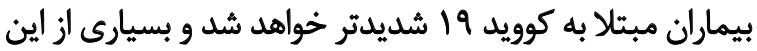

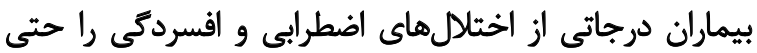

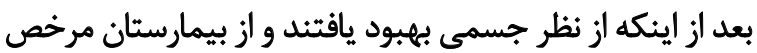

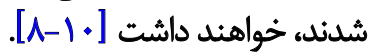

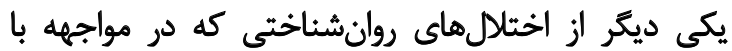

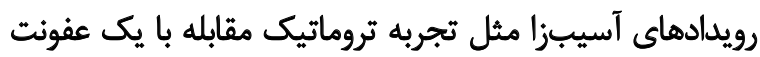

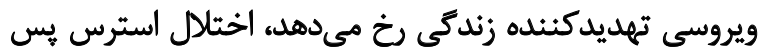

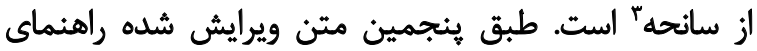

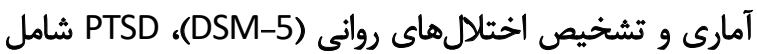

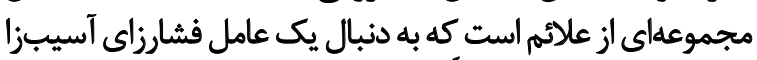

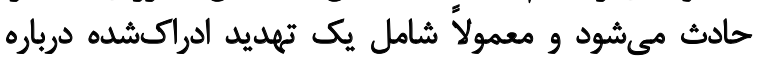

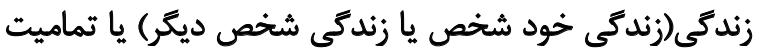

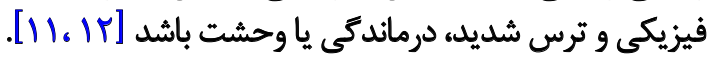
بررسىها نشان دادهاند كه وقتى اييدمي SARS به به به اختلال

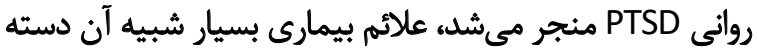

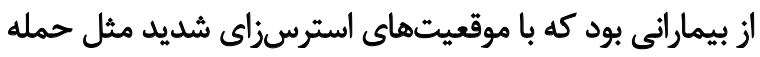

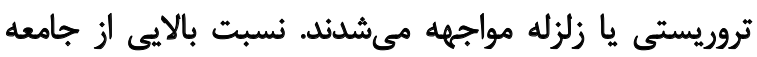

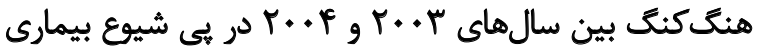

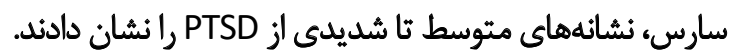
بر اساس نتايج برخى از مطالعات روى سلامت روانى كادر

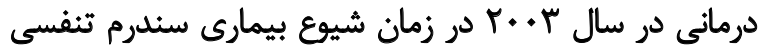

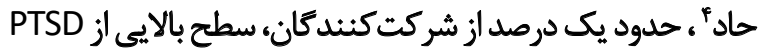

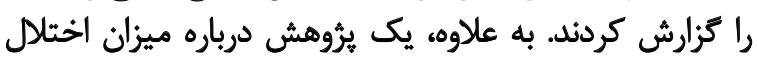

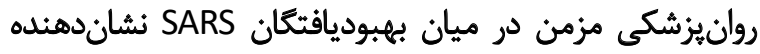

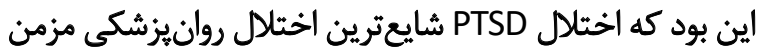

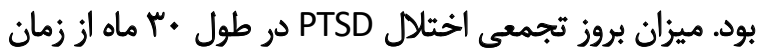

3. Post-Traumatic Stress Disorder (PTSD)

4. Severe Acute Respiratory Syndrome (SARS) dale.

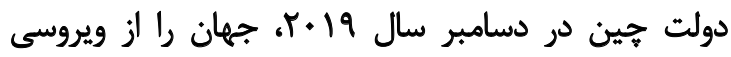

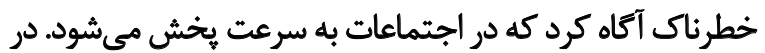

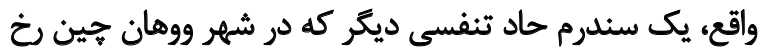

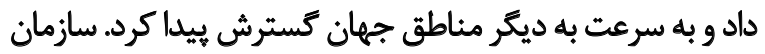

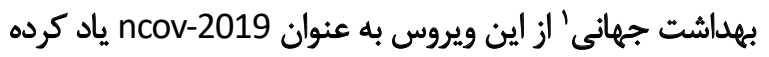

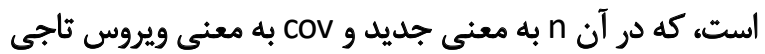

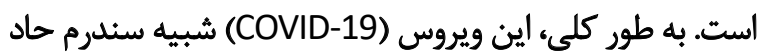

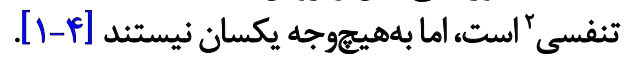
سازمان بهداشت جهانى در 11 مارس، شيوع عفونت كوريد

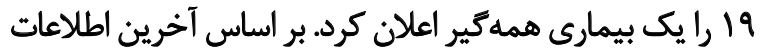

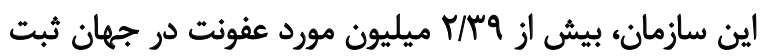

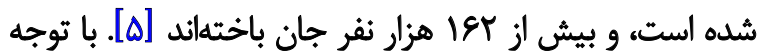

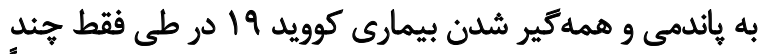

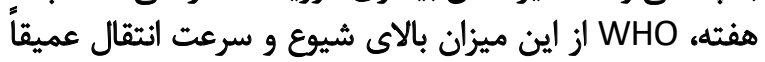

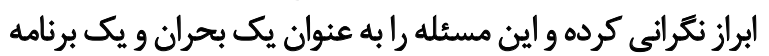

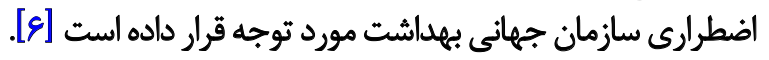
اما در دل هر ناراحتى جسمى، ارتباط محكمى بين مفاهيم

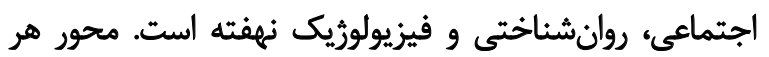

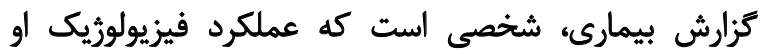

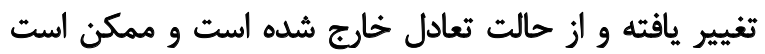

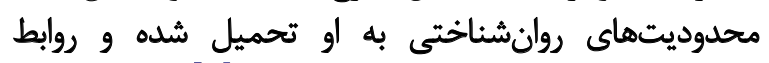

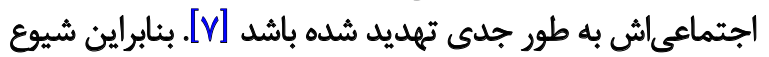

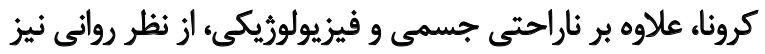

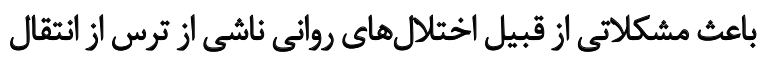

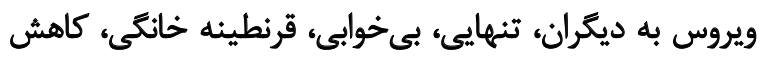

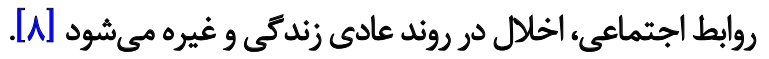

1. World Health Organization (WHO)

2. Middle EastRespiratory Syndrome- Corona Virus (MERS-cov) 
بروسى فيا نشان داده كه مداخله در بحران بايد به صورت يويا

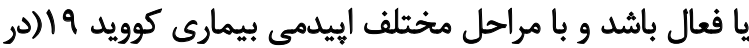

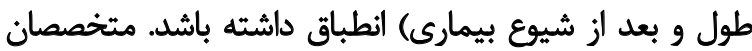

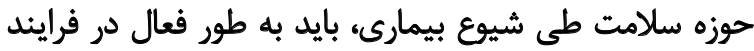

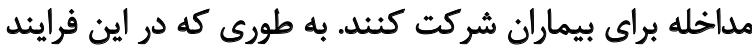

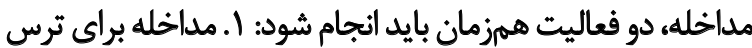

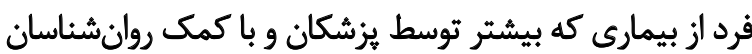

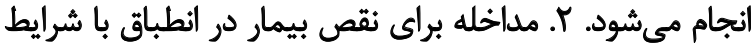

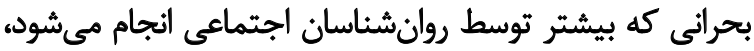

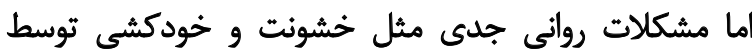

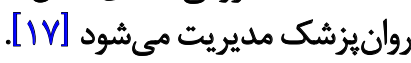

به طور كلى، متخصصان سلامت روان و مددكاران اجتماعى متاني

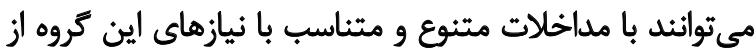

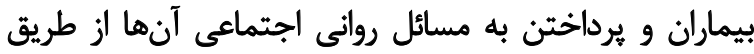

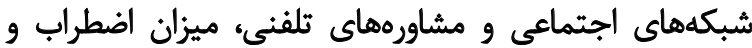

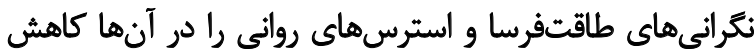

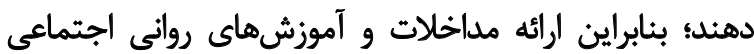

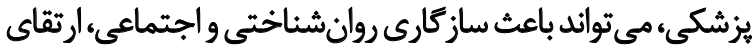

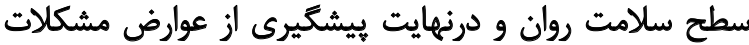

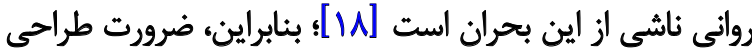

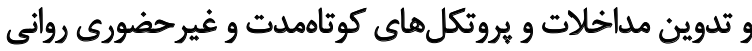

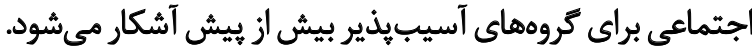

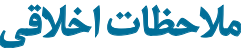

\section{يبروى أز اصول اخلاق يُوهش}

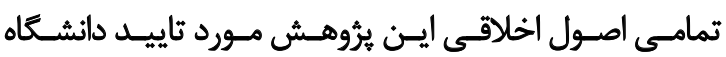

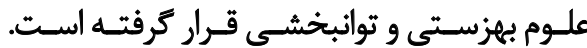

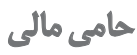

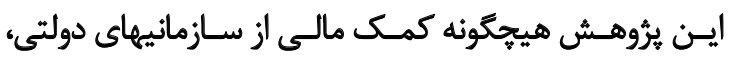

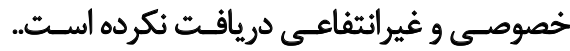

$$
\text { مشاركت نويسندنَان }
$$

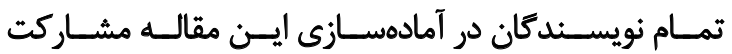
داشــــاند.

$$
\text { ت تعارض مئاقع }
$$

بنابر اظهار نويسنده مسئول، اين مقاله تعارض منافع نداشته

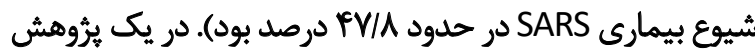

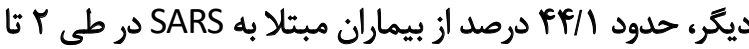

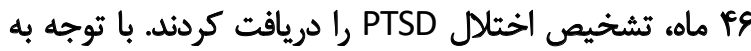

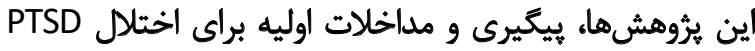

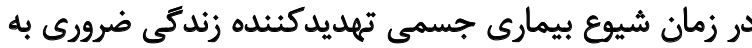

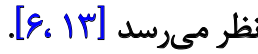

بر اساس يك برؤهش جديد كه توسط سان و همكاران

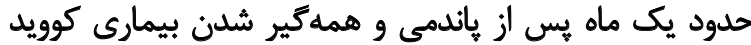

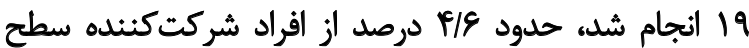

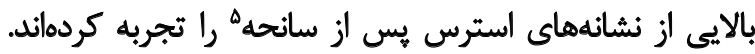

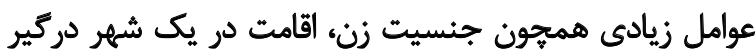

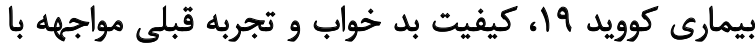

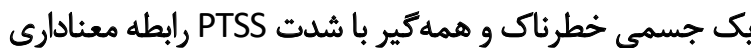

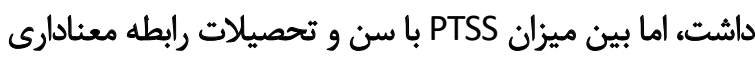

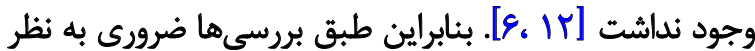

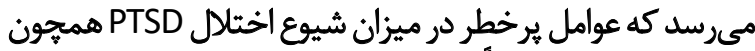

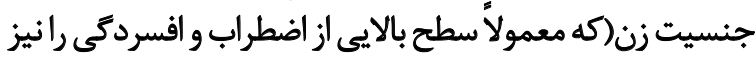

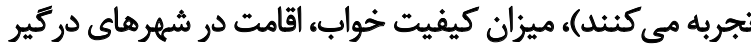

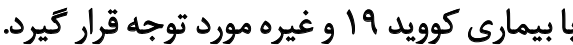

با توجه به احتمال شيوع اختلال PTSD در بيماران كوويد

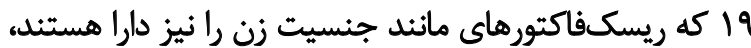

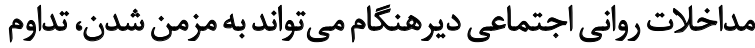

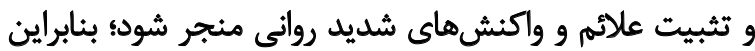

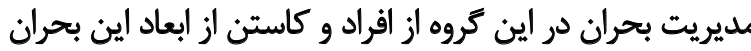

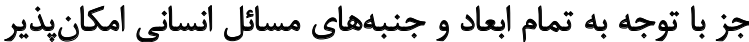

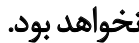
در دوران شرايط بحرانى كوويد 19، اين گروه از بيمارانى كه با باني

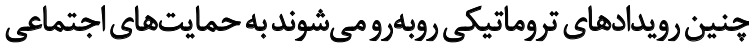

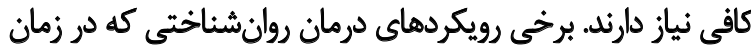

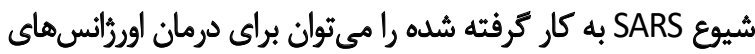

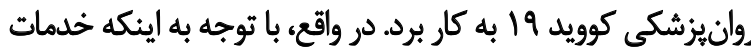

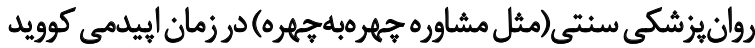

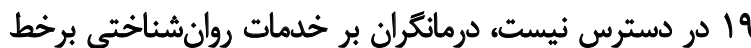
متمركز شدهاند.

جامعه بينالمللى مطالعات استرسهاى تروماتيك؛ و انجمن

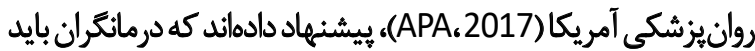

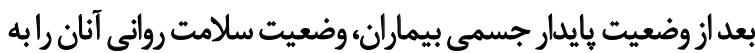

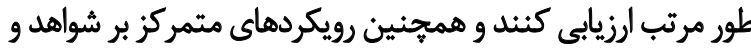

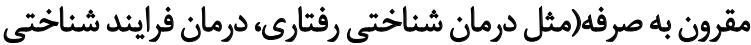

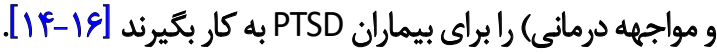

5. Post-Traumatic Stress Symptoms (PTSS)

6. The International Society of Traumatic Stress Studies (ISTSS) 


\section{References}

[1] Wu C, Zheng M, Yang Y, Gu X, Yang K, Li M, et al. Furin: A potential therapeutic target for COVID-19. Iscience. 2020; 23(10):101642. [DOI:10.1016/j.isci.2020.101642] [PMID] [PMCID]

[2] Yamamoto M, Matsuyama S, Li X, Takeda M, Kawaguchi Y, Inoue JI, et al. Identification of nafamostat as a potent inhibitor of Middle East respiratory syndrome coronavirus S protein-mediated membrane fusion using the split-protein-based cell-cell fusion assay. Antimicrobial Agents and Chemotherapy. 2016; 60(11):6532-9. [DOI:10.1128/AAC.01043-16] [PMID] [PMCID]

[3] Arefi MF, Poursadeqiyan M. A review of studies on the COVID-19 epidemic crisis disease with a preventive approach. Work. 2020; 66(4):717-29. [DOI:10.3233/WOR-203218] [PMID]

[4] Poursadeqiyan M, Bazrafshan E, Arefi MF. Review of environmental challenges and pandemic crisis of Covid-19. Journal of Education and Health Promotion. 2020; 9:250. [DOI:10.4103/jehp. jehp_420_20] [PMID] [PMCID]

[5] WHO. Coronavirus Disease (COVID-19) pandemic. Geneva: WHO. https://www.who.int/emergencies/diseases/novelcoronavirus-2019?gclid=EAIaIQobChMIi4bC_Ozt7gIVD-ztCh3kWQUnEAAYASAAEgIsfvD_BwE

[6] Sun L, Sun Z, Wu L, Zhu Z, Zhang F, Shang Z, et al. Prevalence and risk factors of acute posttraumatic stress symptoms during the COVID-19 Outbreak in Wuhan, China. MedRxiv. 2020. [DOI: 10.1101/2020.03.06.20032425]

[7] Dimateo R. Health psychology. [SM. Mousavi Asl, MR. Salari Far, M. Azarbaijani, A. Abbasi, Persian trans.]. Tehran: Samt; 2018. https://samta.samt.ac.ir/content/13628/

[8] Bo H-X, Li W, Yang Y, Wang Y, Zhang Q, Cheung T, et al. Posttraumatic stress symptoms and attitude toward crisis mental health services among clinically stable patients with COVID-19 in China. Psychological Medicine. 2020:1-2. [DOI:10.1017/ S0033291720000999] [PMID] [PMCID]

[9] Huang Y, Zhao N. Generalized anxiety disorder, depressive symptoms and sleep quality during COVID-19 epidemic in China: A web-based cross-sectional survey. Psychiatry Research. 2020; 288:112954. [DOI:10.1101/2020.02.19.20025395]

[10] Qiu J, Shen B, Zhao M, Wang Z, Xie B, Xu Y. A nationwide survey of psychological distress among Chinese people in the COVID-19 epidemic: Implications and policy recommendations. General Psychiatry. 2020; 33(2):e100213. [DOI:10.1136/ gpsych-2020-100213] [PMID] [PMCID]

[11] Firoozabadi A, Asgharnejad Farid AA, Mirzaei J, Shareh H. [Normalization of clinician administered PTSD scale-version 1(caps-1) for psychological effects due to war (Persian)]. Iranian Journal of Psychiatry and Clinical Psychology. 2010; 15(4):334-42. http://ijpcp.iums.ac.ir/article-1-887-en.pdf

[12] Rahnejat AM, Dabagi P, Rabiei M, Taghva A, Valipoor H, Donyavi $\mathrm{V}$, et al. Prevalence of post-traumatic stress disorder caused by war in veterans. Iranian Journal of War and Public Health. 2017; 9(1):15-23. [DOI:10.18869/acadpub.ijwph.9.1.15]

[13] Jiang HJ, Nan J, Lv ZY, Yang J. Psychological impacts of the COVID-19 epidemic on Chinese people: Exposure, post-traumatic stress symptom, and emotion regulation. Asian Pacific Journal of Tropical Medicine. 2020; 13(6):252-9. https://www.apjtm.org/ article.asp?issn=1995-7645; year=2020; volume=13;issue=6; ;page= 252; epage $=259$;aulast=Jiang

[14] Shi W, Hall BJ. What can we do for people exposed to multiple traumatic events during the coronavirus pandemic?. Asian Journal of Psychiatry. 2020;51:102065. [DOI:10.1016/j.ajp.2020.102065] [PMID] [PMCID]

[15] Zhang F, Shang Z, Ma H, Jia Y, Sun L, Guo X, et al. High risk of infection caused posttraumatic stress symptoms in individuals with poor sleep quality: A study on influence of Coronavirus disease (COVID-19) in China. MedRxiv. 2020. [DOI:10.1101/2020 .03.22.20034504]

[16] Zhu Y, Chen L, Ji H, Xi M, Fang Y, Li Y. The risk and prevention of novel coronavirus pneumonia infections among inpatients in psychiatric hospitals. Neuroscience Bulletin. 2020; 36(3):299-302. [DOI:10.1007/s12264-020-00476-9] [PMID] [PMCID]

[17] Zhang J, Wu W, Zhao X, Zhang W. Recommended psychological crisis intervention response to the 2019 novel coronavirus pneumonia outbreak in China: A model of West China Hospital. Precision Clinical Medicine. 2020; 3(1):3-8. [DOI:10.1093/pcmedi/pbaa006] [PMCID]

[18] Javadi SMH, Arian M, Qorbani-Vanajemi M. The need for psychosocial interventions to manage the coronavirus crisis. Iranian Journal of Psychiatry and Behavioral Sciences. 2020; 14(1):e102546. https://sites.kowsarpub.com/ijpbs/articles/102546.html 